\title{
Evaluating the Anxiety and Depression Status of Prostate Cancer Patients whose Operations were Postponed due to the COVID-19 Pandemic
}

\author{
Yalcin Kizilkan ${ }^{1}$, Samet Senel ${ }^{1}$, Ali Yasin Ozercan ${ }^{1}$, Melih Balci ${ }^{1}$, Unsal Eroglu ${ }^{1}$, Binhan \\ Kagan Aktas ${ }^{1}$, Suleyman Bulut ${ }^{1}$, Cüneyt Özden ${ }^{1}$, and Altug Tuncel ${ }^{1}$ \\ ${ }^{1}$ Ankara City Hospital
}

December 18, 2020

\begin{abstract}
Aim: In this study, we aimed to evaluate the anxiety and depression status of prostate cancer (PCa) patients whose planned operations in the urology clinic of our hospital, which is serving as a pandemic hospital in Turkey have been postponed due to the coronavirus disease 2019 pandemic. Methods: This survey study was conducted at urology clinic of Ankara City Hospital between March 1 and June 1, 2020 and included 24 male patients who agreed to answer the questionnaires (State-Trait Anxiety Inventory [STAI] I and II and Beck Depression Inventory [BDI]). Demographical and clinical data (age, time since diagnosis, total serum prostate specific antigen (PSA) levels, risk groups according to the D'Amico classification system, smoking, alcohol habitus, major surgical history, and comorbidities) of the patients were collected from hospital software. Results: The mean STAI-I score of the patients $(46.7 \pm 1.4[44-49])$ was significantly higher than their STAI-II score $(41.7 \pm 2.4[39-47])(\mathrm{p}<$ 0.001). The negative correlation between the decrease in age and STAI-I score was found to be statistically significant $(\mathrm{r}=$ $0.439, \mathrm{p}<0.05)$. The mean BDI score of the patients was $4.3 \pm 3.2(0-13)$, which was compatible with mild depression. There was no statistically significant difference between the time elapsed from diagnosis, PSA levels, smoking and alcohol habitus, major surgical history and comorbidity status, and STAI-I, STAI-II, and BDI scores ( $\mathrm{p}>0.05$ ). Conclusion: Prostate cancer patients with postponed operations should be guided properly in order to manage their anxiety status especially young patients.
\end{abstract}

Evaluating the Anxiety and Depression Status of Prostate Cancer Patients whose Operations were Postponed due to the COVID-19 Pandemic

\section{Abstract}

Aim: In this study, we aimed to evaluate the anxiety and depression status of prostate cancer (PCa) patients whose planned operations in the urology clinic of our hospital, which is serving as a pandemic hospital in Turkey have been postponed due to the coronavirus disease 2019 pandemic.

Methods: This survey study was conducted at urology clinic of Ankara City Hospital between March 1 and June 1, 2020 and included 24 male patients who agreed to answer the questionnaires (State-Trait Anxiety Inventory [STAI] I and II and Beck Depression Inventory [BDI]). Demographical and clinical data (age, time since diagnosis, total serum prostate specific antigen (PSA) levels, risk groups according to the D'Amico classification system, smoking, alcohol habitus, major surgical history, and comorbidities) of the patients were collected from hospital software.

Results: The mean STAI-I score of the patients (46.7 \pm 1.4 [44-49]) was significantly higher than their STAIII score $(41.7 \pm 2.4[39-47])(\mathrm{p}<0.001)$. The negative correlation between the decrease in age and STAI-I score was found to be statistically significant $(\mathrm{r}=0.439, \mathrm{p}<0.05)$. The mean BDI score of the patients was $4.3 \pm 3.2(0-13)$, which was compatible with mild depression. There was no statistically significant difference 
between the time elapsed from diagnosis, PSA levels, smoking and alcohol habitus, major surgical history and comorbidity status, and STAI-I, STAI-II, and BDI scores ( $\mathrm{p}>0.05)$.

Conclusion: Prostate cancer patients with postponed operations should be guided properly in order to manage their anxiety status especially young patients.

Keywords: Anxiety; COVID-19; Depression; Postponed operations; Prostate cancer

\section{What is known?}

The diagnosis of PCa itself negatively affects the mental well-being and quality of life.

Postponing the operations of patients diagnosed with cancer may increase the anxiety levels.

\section{What our study adds?}

This is the first study in the literature which evaluated the anxiety and depression levels in urooncology patients whose operation was postponed because of the COVID-19 pandemic.

The patients' state anxiety levels are significantly higher than the trait anxiety level, and the state anxiety level is more pronounced in young patients.

\section{Introduction}

The coronavirus disease 2019 (COVID-19), whose first cases were noted in Wuhan, China in December 2019 , is the third coronavirus epidemic after severe acute respiratory syndrome and Middle East respiratory syndrome. ${ }^{1}$ The epidemic quickly spread worldwide from the moment the first diagnoses were established and the disease affected people from all nations; consequently, it was deemed a pandemic by the World Health Organization on March 11, 2020. On the same day, the first case of COVID-19 was reported by the Ministry of Health in Turkey. ${ }^{2}$

Surgical operations have been delayed according to a specific algorithm worldwide and urological procedures in Turkey have been limited to urgent condition and selected oncological surgeries in accordance with available guidelines for COVID-19 treatments. ${ }^{3}$ Included in the limited surgeries is the prostate cancer (PCa) surgery. The diagnosis of PCa itself negatively affects the mental well-being and quality of life of patients, and postponing the operations of patients diagnosed with cancer for various reasons may increase the anxiety levels of the patients. ${ }^{4}$ Therefore, we aimed to evaluate the anxiety and depression status of PCa patients whose robot-assisted laparoscopic radical prostatectomy (RALRP) operations haves been postponed in the urology clinic of our hospital, which is serving as a pandemic hospital in Turkey during the COVID-19 pandemic.

\section{Materials and Methods}

This is a survey study which was conducted at urology clinic of our hospital between March 1 and June 1, 2020. A total of 39 patients diagnosed with PCa and scheduled for RALRP in this period, whose operations were postponed because of the COVID-19 pandemic, were identified. Twenty-four male patients who approved to answer the questionnaires (State-Trait Anxiety Inventory [STAI] I and II and Beck Depression Inventory [BDI]) were included in the study. This study was approved by our institute's local ethic committee (Approval number: E1-20-1016) and was performed in accordance with the ethical standards laid down in the 1964 Declaration of Helsinki. Informed consent forms were obtained from all patients.

In our hospital, we use D'Amico classification to determine risk group for PCa. According to the D'Amico classification, patients with PSA value less than $10 \mathrm{ng} / \mathrm{mL}$, Gleason scores of 6 , and stage cT1-T2a were evaluated to be in the low-risk group; patients with a PSA values ranging between 10-20 ng/mL, Gleason scores of 7 , and stage cT2b were evaluated to be in the medium-risk group; and patients with a PSA values exceeding $20 \mathrm{ng} / \mathrm{mL}$, Gleason scores of 8 and above, and stage cT2c and above were evaluated to be in the high-risk group. ${ }^{5}$ While patients from the high-risk group were operated upon, the operations of the patients in the low-to-medium risk groups were postponed. 
The patients were subjected to the STAI-I and II to assess their state and trait anxiety together with the BDI to assess their state of depression. The STAI is one of the most commonly used anxiety measurement methods for psychological research. Although numerous methods are available to assess anxiety, STAI is considered as the standard method. STAI-I status assesses a person's instantaneous anxiety level and STAIII persistency evaluates the person's overall anxiety level. STAI includes two separate scales, comprising a total of 40 questions. A positive correlation exists between the score level and anxiety level. The lowest and highest scores on the scale are 19 and 80, respectively. ${ }^{6}$ The Beck Depression Inventory is used to measure the level of depression in the participants. This scale comprises 21 questions to be answered on the basis of the preceding week. Each question includes 4 options as an answer and the options are listed such that they express depression with increasing severity. In the score obtained on the scale, 0-9 points signify minimal depression, 10-16 points signify mild depression, 17-29 points signify moderate depression, and 30-63 points signify severe depression. ${ }^{7}$ These questionnaires have validated Turkish versions, which were performed by the psychiatric societies of our country.

Demographical and clinical data (age, time since diagnosis, total serum prostate specific antigen (PSA) levels, risk groups according to the D'Amico classification system, smoking, alcohol habitus, major surgical history, and comorbidities) of the patients were collected from hospital software.

In this study, STAI I-II and BDI questionnaires were given to all patients and the patients were asked to choose the most appropriate answer for themselves. The results were saved in the data pool. None of the authors received any type of financial or nonfinancial support that could be considered a potential conflict of interest regarding the manuscript or its submission.

\section{Statistical Analysis}

Coding and statistical analyses of data were performed on the computer, using the Statistical Package for the Social Science 22.0 software (SPSS, Inc., Chicago, IL, USA). Descriptive statistical data for continuous variables were expressed as mean, median, standard deviation, percentage, minimum, and maximum values. The Mann-Whitney U test, the Wilcoxon test, and Spearman's correlation analysis were used to analyze and compare the data. In all analyses, the level of statistical significance was considered to be 0.05 .

\section{Results}

The mean age of the patients included the study was $64.8 \pm 7.5$ (51-77) years, the mean time since diagnosis was $78.8 \pm 21.9(48-136)$ days, and the mean total serum PSA level was $9.8 \pm 5.5(4.1-22) \mathrm{ng} / \mathrm{mL}$. The demographical data of the patients are listed in Table 1. Of the 24 patients whose operations were postponed, $14(58.3 \%)$ patients were in the low-risk group and $10(41.7 \%)$ patients were in the medium-risk group. Fourteen patients have Gleason 3+3, eight patients have Gleason $3+4$ and 2 patients have Gleason $4+3$ prostatic adenocarcinoma.

The mean STAI-I score of the patients $(46.7 \pm 1.4$ [44-49]) was significantly higher than their STAI-II score $(41.7 \pm 2.4[39-47])(\mathrm{p}<0.001)$. The negative correlation between the decrease in age and STAI-I score was found to be statistically significant $(\mathrm{r}=0.439, \mathrm{p}<0.05)$. The mean BDI score of the patients was 4.3 $\pm 3.2(0-13)$, which was compatible with mild depression. There was no statistically significant difference between the time elapsed from diagnosis, PSA levels, smoking and alcohol habitus, major surgical history and comorbidity status, and STAI-I, STAI-II, and BDI scores (p>0.05) (Table 2 and 3).

\section{Discussion}

While elective surgeries have been postponed in most centers due to the COVID-19 pandemic, emergency cases and oncological surgeries are recommended to be performed with some precautions. ${ }^{8}$ Therefore, we evaluated the anxiety and depression status of PCa patients whose RALRP operations were postponed in our hospital, which is one of the most important pandemic centers in Turkey. Through our study, we obtained some important results. The patients' state anxiety levels are significantly higher than the trait anxiety level, and the state anxiety level is more pronounced in young patients. 
In the European Urology Guidelines, $\mathrm{PCa}$ is recommended to be divided into risk groups according to the D'Amico classification system; moreover, its treatment must be classified. ${ }^{9}$ According to this classification system, radical prostatectomy (RP) remains the most valid treatment method for low- and medium-risk PCa patients with a 10-year life expectancy; in addition, RP is recommended as part of multimodal therapy in high-risk PCa. ${ }^{10,11}$ It has been emphasized that during the COVID-19 pandemic period, RP treatment is recommended to be postponed in patients with low- and medium-risk PCa to the post-epidemic period; moreover, the surgery of selected high-risk PCa patients may also be postponed as delaying surgical treatment for up to 12 months does not affect operation results and cancer specific survival even in high-risk patients. ${ }^{12}$ In the literature, different results are highlighted in a limited number of studies that have examined the anxiety and depression states in patients whose operations were postponed. The study conducted by Frey et al. ${ }^{13}$, wherein the impact of the COVID-19 epidemic on cancer treatments and quality of life of 555 patients diagnosed with ovarian cancer was evaluated using the Hospital Anxiety and Depression scale and the Cancer Anxiety Scale, reported the treatment of 28 (11\%) out of 225 patients who needed chemotherapy or surgical treatment and whose operations were postponed. Therein, $23.4 \%$ of the participating patients had anxiety and $7.6 \%$ of the patients had depression. In addition, Frey et al. emphasized that the anxiety rate ranged between $13.9 \%-48.1 \%$ and the depression rate ranged between $5 \%-33.7 \%$ in the non-epidemic period; furthermore, no difference was observed between the two periods in terms of anxiety and depression rates. In another study ${ }^{14}$ conducted before the pandemic, the depression status of 390 patients on the waiting list for renal transplantation were evaluated and the BDI was applied to the patients four times: at the time when they entered the list, in the $12^{\text {th }}$ waiting month, in the $24^{\text {th }}$ waiting month, and in the $3^{\text {rd }}$ month after transplantation. According to the test results, the mean BDI score was $5.5 \pm 5.1$ (mild depression) and the rate of patients diagnosed with depression was $8.7 \%$ at the time they entered the list, which increased to $12.4 \%$ in the $12^{\text {th }}$ waiting month, and on to $16.7 \%$ in the $24^{\text {th }}$ waiting month. After transplantation, this rate decreased to $2.3 \%$. In a recent study where Karahan et al. ${ }^{15}$ evaluated the effects of postponing abdominal, endocrine, and orthopedic surgeries on patients' anxiety levels for any reason regardless of the COVID-19 epidemic and compared 50 patients with postponed surgery and 50 patients whose surgeries were not postponed, it was reported that while there was no significant difference between groups in terms of state anxiety levels, the level of persistent trait anxiety in the group whose operation was deferred was statistically significantly higher. This result was interpreted by the authors in the sense that the high trait anxiety scores of the patients whose surgical intervention was postponed might be reflection of personal characteristics. In Rodler et al's study ${ }^{16}, 92$ patients with advanced genitourinary cancers were surveyed about their perceptions of anxiety of COVID-19 and cancer. $56.5 \%$ of the patients were more anxious about cancer than COVID-19, 26.1\% of them were more anxious about COVID-19 than cancer. They also declared that despite the pandemic, cancer remained the main health issue for these patients and they were not willing to compromise on their treatment.

To the best of our knowledge, no study has been conducted to investigate the effects of this situation on anxiety and depression levels in urooncology patients whose surgical treatment was postponed because of the COVID-19 pandemic. While the strength of our study is that it is the first study on this topic, its weakness is that the anxiety and depression levels of the patients were not known before the pandemic process. However, considering that this process is unpredictable, this discrepancy seems to be acceptable. In a study that examined the factors associated with the anxiety and depression status of patients with prostate cancer, it was reported that PSA level, patient age, and number of comorbidities are not related to anxiety and depression ${ }^{17}$. In the present study, we did not identify any relationship between PSA levels, history of smoking and alcohol habitus, history of major surgery and comorbidity, and STAI-I, STAI-II, and BDI scores. According to the results of our study, the STAI-I levels of patients whose RALRP surgeries were postponed due to the COVID-19 pandemic were significantly higher than those of STAI-II. In addition, according to the BDI, the mean score of the patients was $4.3(0-13)$, which signifies mild depression. This result can be interpreted in the sense that the pandemic and/or the postponement of the operation because of the pandemic caused additional anxiety in patients. The STAI-I levels in younger patients were statistically significantly higher than those in the elderly population. The higher awareness level in young patients might explain why young patients have higher anxiety levels in our study. 
Because of the patient population in our study belongs to a narrow cross-sectional timeframe, the number is small as expected. This low number is a limitation of our study.

\section{Conclusion}

The results of our study showed that the state anxiety levels of PCa patients with postponed RALRP operations are significantly higher than the trait anxiety levels. In addition, in young patients, the level of anxiety is higher than elderly patients. Patients with postponed operations should be well informed and supported through the process. Counseling may help patients with high anxiety levels.

\section{Conflict of interest}

The authors declare that they have no conflict of interest.

\section{Funding}

No funding sources.

\section{Author contribution}

Dr YK had the primary responsibility for writing the manuscript. Dr SS, Dr AYO, Dr MB and Dr UE participated in protocol development, preliminary data analysis. Dr BKA, Dr SB, Dr CO and Dr AT participated in the development of the protocol and analytical framework for the study and the revision of the manuscript.

\section{Data accessibility statement}

The data that support the findings of this study are available from the corresponding author upon reasonable request.

\section{References}

Guarner J. Three Emerging Coronaviruses in Two Decades. Am J Clin Pathol. 2020 Mar 9;153(4):420-421.

Soytas M, Boz MY, Guzelburc V, et al. Analysis of patients undergoing urological intervention amid the COVID-19: experience from the pandemic hospital. Int Urol Nephrol. 2020 Nov;52(11):2059-2064.

Ribal MJ, Cornford P, Briganti A, et al. EAU Section Offices and the EAU Guidelines Panels. European Association of Urology Guidelines Office Rapid Reaction Group: An Organisation-wide Collaborative Effort to Adapt the European Association of Urology Guidelines Recommendations to the Coronavirus Disease 2019 Era. Eur Urol . 2020 Jul;78(1):21-28.

Lehto US., Tenhola H, Taari K, Aromaa A. Patients' perceptions of the negative effects following different prostate cancer treatments and the impact on psychological well-being: a nationwide survey. $\mathrm{Br} J \mathrm{Cancer}$ 2017;116(7):864-873.

D'Amico AV, Whittington R, Malkowics SB, et al. Biochemical outcome after radical prostatectomy, external beam radiotion therapy or interstitial radiation therapy for clinically localized prostate cancer.JAMA 1998; 280: 969-974.

Marteau TM, Bekker H. The development of a six-item short form of the state scale of the Spielberg StateTrait Anxiety Inventory (STAI).Br J Clin Psychol 1992;31: 301-36.

Whisman MA, Richardson ED. Normative Data on the Beck Depression Inventory-Second Edition (BDI-II) in College Students. J Clin Psychol. 2015 Sep;71(9):898-907.

Ficarra V, Novara G, Abrate A, et al. Practice during the COVID-19 pandemic. Minerva Urol Nefrol. 2020 Jun;72(3):369-375. 
Mottet N, van den Bergh RCN, Briers E, et al. EAU-EANM-ESTRO-ESUR-SIOG Guidelines on Prostate Cancer-2020 Update. Part 1: Screening, Diagnosis, and Local Treatment with Curative Intent. Eur Urol. 2020 Nov 7:S0302-2838(20)30769-7.

Hamada R, Nakashima J, Ohori M, et al. Preoperative predictive factors and further risk stratification of biochemical recurrence in clinically localized high-risk prostate cancer. Int J Clin Oncol 2015; 21(3): 595-600.

Aktas BK, Ozden C, Bulut S, et al. Evaluation of Biochemical Recurrence-free Survival after Radical Prostatectomy by Cancer of the Prostate Risk Assessment Post-Surgical (CAPRA-S) Score. Asian Pac J Cancer Prev 2015; 16(6): 2527-2530.

Stensland KD, Morgan TM, Moinzadeh A, et al. Considerations in the Triage of Urologic Surgeries During the COVID-19 Pandemic. Eur Urol. 2020 Jun;77(6):663-666.

Frey MK, Ellis AE, Zeligs K, et al. Impact of the coronavirus disease 2019 pandemic on the quality of life for women with ovarian cancer.Am J Obstet Gynecol. 2020 Nov;223(5):725.e1-725.e9

Corruble E, Durrbach A, Charpentier B, et al. Progressive increase of anxiety and depression in patients waiting for a kidney transplantation. Behav Med. 2010;36(1):32-36.

Karahan E, Celik S, Zaman F. The Reasons for Cancellation of Elective Surgical Interventions and Its Effect on Patient Anxiety Level.CBU-SBED, 2020, 7(2): 123 -129.

Rodler S, Apfelbeck M, Schulz GB, et al. Telehealth in Uro-oncology Beyond the Pandemic: Toll or Lifesaver? Eur Urol Focus . 2020 Sep 15;6(5):1097-1103.

Dale W, Bilir P, Han M, Meltzer D. The role of anxiety in prostate carcinoma: a structured review of the literature. Cancer. 2005;104: 467-78.

\section{Hosted file}

Table 1.pdf available at https://authorea.com/users/383695/articles/499545-evaluatingthe-anxiety-and-depression-status-of-prostate-cancer-patients-whose-operations-werepostponed-due-to-the-covid-19-pandemic

\section{Hosted file}

Table 2.pdf available at https://authorea.com/users/383695/articles/499545-evaluatingthe-anxiety-and-depression-status-of-prostate-cancer-patients-whose-operations-werepostponed-due-to-the-covid-19-pandemic

\section{Hosted file}

Table 3.pdf available at https://authorea.com/users/383695/articles/499545-evaluatingthe-anxiety-and-depression-status-of-prostate-cancer-patients-whose-operations-werepostponed-due-to-the-covid-19-pandemic 